\title{
Biomechanical effects of passive hip springs during walking
}

\section{Journal Article}

Author(s):

Haufe, Florian (D); Wolf, Peter (D); Riener, Robert; Grimmer, Martin

Publication date:

2020-01-02

Permanent link:

https://doi.org/10.3929/ethz-b-000382233

\section{Rights / license:}

Creative Commons Attribution-NonCommercial-NoDerivatives 4.0 International

\section{Originally published in:}

Journal of Biomechanics 98, https://doi.org/10.1016/j.jbiomech.2019.109432 


\title{
Biomechanical Effects of Passive Hip Springs During Walking
}

\author{
Florian L. Haufe ${ }^{\mathrm{a}, *}$, Peter Wolf ${ }^{\mathrm{a}}$, Robert Riener ${ }^{\mathrm{a}, \mathrm{b}}$, Martin Grimmer $^{\mathrm{c}}$ \\ ${ }^{a}$ Sensory-Motor Systems (SMS) Lab, Institute of Robotics and Intelligent Systems \\ (IRIS), ETH Zurich, Switzerland \\ ${ }^{b}$ Spinal Cord Injury Center, Balgrist University Hospital, Medical Faculty, University of \\ Zurich, Switzerland \\ ${ }^{c}$ Institute for Sports Science, TU Darmstadt, Germany
}

\begin{abstract}
Passive spring-like structures can store and return energy during cyclic movements and thereby reduce the energetic cost of locomotion. That makes them important components of the human body and wearable assistive devices alike. This study investigates how springs placed anteriorly across the hip joint affect leg joint angles and powers, and leg muscle activities during level walking at 0.5 to $2.1 \mathrm{~m} / \mathrm{s}$.

We hypothesized that the anterior hip springs (I) load hip extension, (II) support hip flexion and (III) affect ankle muscle activity and dynamics during walking. Effects at the ankle were expected because hip and ankle redistribute segmental power in concert to achieve forward progression.

We observed that the participants' contribution to hip power did not increase during hip extension as the spring stored energy. Simultaneously, the activities of plantarflexor muscles that modulate energy storage in the Achilles tendon were reduced by $28 \%$ (gastrocnemius medialis) and $9 \%$ (soleus). As the spring returned energy with the onset of hip flexion, the participants' contribution to hip power was reduced by as much as $23 \%$. Soleus activity before push-off increased by up to $9 \%$.

Instead of loading hip extension, anterior hip springs seem to store and return parts of the energy normally exchanged with the Achilles tendon. Thereby, the springs support hip flexion but may reduce elastic energy stor-
\end{abstract}

\footnotetext{
* Corresponding author

Email address: florian.haufe@hest.ethz.ch (Florian L. Haufe)
} 
age in and hence recoil from the Achilles tendon. This interaction should be considered during the design and simulation of wearable assistive devices as it might - depending on user characteristics - enhance or diminish their overall functionality.

Keywords: physiology, exoskeleton, exosuit, gait

\section{Introduction}

Passive energy storage and return has long been recognized as one of the central mechanisms for minimizing the energetic cost of terrestrial locomotion (Cavagna et al., 1977). This mechanism relies on the deformation of 5 passive structures such as tendons or ligaments to elastically store energy when the movement direction of body segments periodically changes. The stored energy can be returned to accelerate body segments into their next movement phases instead of being dissipated at every inflection point, potentially reducing the required active energy input.

Inspired by this, researchers have aimed to improve energy efficiency, to reduce control complexity, and to adapt stiffness by integrating elastic elements, or more figuratively, springs, into prostheses (Shepherd and Rouse, 2017) and orthoses (Blaya and Herr, 2004, Bregman et al., 2012, Collins et al., 2015, Ferris et al., 2006, Shamaei et al., 2015).

While hip, knee and ankle joints all potentially allow for the addition of parallel springs, previous work has been primarily focused on designs crossing the ankle joint (Blaya and Herr, 2004, Bregman et al., 2012, Collins et al., 2015, Ferris et al., 2006). The ankle joint is of central interest due to its large contribution to overall positive power during walking, clear separation of dissipative and propulsive phases, and its role in overall leg stiffness modulation (Hobara et al., 2010). In addition, the shank and foot allow for convenient circumferential attachment of wearable robots with effective force transmission, at least for healthy users (Collins et al., 2015).

In contrast, net power at the knee joint is predominantly negative during those at the hip or ankle. Muscles acting at the knee joint might hence primarily contribute to limb stiffness modulation or deceleration rather than to body acceleration (Farris and Sawicki, 2012), making the knee less attractive for cyclic energy storage and return. 
More akin to the ankle, the dynamics of the hip joint do potentially allow for passive energy storage and return during walking. One measure that is often analyzed in this context is the quasi-stiffness of a joint, or the slope of the moment-angle relationship during a dynamic movement (Rouse et al., 2013). Previous work analyzing the quasi-stiffness of the human hip joint has extension offers an attractive opportunity for "spring-like" external assistance (Shamaei et al., 2013). Here, an anteriorly placed spring could store energy in the phase preceding maximum hip extension while effectively supporting leg deceleration, and return that energy afterwards to support hip flexion. 40 Comparable spring setups have already been shown to improve gait function after stroke (Carda et al., 2012). In addition, findings from previous research suggest that in healthy humans, hip flexion during walking is largely caused by passive elastic forces from deformed soft tissue, and only to a lesser extent by active muscle force (Simonsen et al., 2012). In this light, passive springs are an attractive concept to support hip flexion in a physiological and welltolerated manner. For example, it was recently shown that a spring applying torque as a linear function of the difference between left and right hip angle reduces the metabolic cost of running in healthy users (Nasiri et al., 2018).

However, so far, springs across the hip joint have been primarily described as part of simulation studies (e.g. Cahill et al. (2018)), in studies with a clinical focus that did not include a biomechanical analysis (Carda et al., 2012, Sutliff et al., 2008), or as part of multi-joint orthoses (e.g. Schmidt et al. (2017), Walsh et al. (2007)). While the latter often achieved better outcomes than isolated hip springs during real-world applications, the clear discrimination of effects stemming from the hip springs and those stemming from other components is difficult. As of now, little empirical data on the isolated biomechanical effects of springs anteriorly placed across the hip joint is available. With this study, we aimed to characterize these effects during healthy human walking.

60 Following previous literature, we defined the gait cycle (GC) from heelstrike $(0 \%)$ to the subsequent heelstrike of the same foot $(100 \%)$. Regarding the hip spring, two main phases can be defined: the "(spring) energy storage phase" in which spring power is negative and energy is stored, and the "(spring) energy return phase", where spring power is positive and energy is returned (see Figure 1). During the energy storage phase, the spring initially counteracts the hip extension moment, but then works in line with the flexion moment that decelerates the thigh and finally supports hip flexion 
during the energy return phase. For the purpose of our analysis, we consider hip joint power as the "total" sum composed of "biological" and "spring" contributions. Here, the prefix "biological" refers to the participant's contribution.

Within this framework, we formulated three main hypotheses.

Hypothesis I: A passive linear spring that is anteriorly placed at the hip increases the required biological contribution to hip extension during level 75 walking.

We assumed that an increased biological contribution to hip extension would show as an increase of the mean positive biological hip power during energy storage phase as the spring is extended. This would also be reflected in increased hip extensor muscle activities.

80 Hypothesis II: The hip spring reduces the required biological contribution to hip flexion during level walking.

We assumed that a reduced biological contribution to hip flexion would show as a reduction of the mean positive biological hip power during energy return phase as the spring shortens and returns energy. Throughout the gait cycle, we did not expect changes in the total hip power because we assumed that biological power would be modulated to compensate for the contribution of the spring.

Hypothesis III: The hip spring affects the activity of muscles acting on the ankle joint and ankle dynamics during level walking.

During walking, muscles spanning the hip or ankle joint work in a concerted manner to redistribute segmental power and thereby achieve forward progression of the legs and the trunk (Neptune et al., 2004). As a response to the added spring stiffness at the hip, we expect to see a change in the quasi-stiffness of the ankle joint. Based on the work of Latash and Zatsiorsky (1993), we followed that such a change would be evident in the EMG activity of plantarflexor and dorsiflexor muscles.

Bidirectional transfer effects between the hip and the ankle joint have been observed before: In a study by Lenzi et al. (2013), an active hip exoskeleton has been shown to affect plantarflexor muscle activation, while Mooney and Herr (2016) have reported that an active ankle exoskeleton changed the hip dynamics during walking. 
Given the short anticipated overlap between the onset of the energy return phase at maximum hip extension (approx. 54\% GC) and toe off (approx. $61 \%$ GC), we do not expect that ankle plantarflexion can be supported by the spring. Hence, the first half of the GC (heel strike to push-off), and, in particular, the spring energy storage phase is left as the region of interest.

To evaluate our hypotheses, a wearable, functional demonstrator with minimal weight and footprint was designed and built from textile and elastomeric materials. The underlying rationale here was to resemble the ideal superposition of an assistive force - as performed in simulations, e.g. Dembia et al. (2017) - as closely as possible, and avoid compensatory movements induced by discomfort or added mass. Our analysis is intended to enhance the understanding of human-robot interaction in wearable robots, as well as to inform the development of combined active/passive actuation schemes.

\section{Methods}

\subsection{Participants}

Nine healthy males, age $28(23-38)$ yrs, height $177(170-183) \mathrm{cm}$, mass $71(61-83) \mathrm{kg}$, mean (range), were recruited as a local convenience sample and participated in the study after they gave their informed consent. The study design and protocol were approved by the institutional review board of ETH Zurich (EK 2018-N-11).

\subsection{Design of Functional Demonstrator}

Spring elements were made from commercially available elastomer resistance band (TheraBand Professional Latex Resistance Band Gold, Performance Health, USA). The resistance band was cut into segments with a length of $18 \mathrm{~cm}$ (width of $12.5 \mathrm{~cm}$ as delivered). Single- and double-stiffness spring elements were manufactured by sewing together one or two parallel pieces of resistance band and four industrial grade hook and loop fastener patches ( $5 \mathrm{~cm}$ by $12.5 \mathrm{~cm}$, Velcro BVBA, UK) at each end of both sides, leaving an effective slack length of $8 \mathrm{~cm}$ between the patches (see Figure 2). The final spring arrangement had an experimentally-determined linear tensile stiffness of $801 \mathrm{~N} / \mathrm{m}$ for each leg for the single- and of $1602 \mathrm{~N} / \mathrm{m}$ per leg for the double-stiffness spring (see Supplementary Information Figure 1). In compression direction, the springs had no measurable stiffness.

A custom-made upper body vest and two modified commercial thigh braces (MyoActiv Sport Thigh Support, BORT GmbH, Germany) were used 
to symmetrically anchor the spring elements anteriorly across each hip joint. On both the vest and the two thigh braces, hook and loop patches were attached to anchor the spring elements. At the distal end of the brace, a high-stiffness hook fastener band was circumferentially attached to achieve a conical anchoring on the participants thighs. Springs were attached between the trunk and thigh anchor points with a pretension of $3.5 \mathrm{~cm}$ which corresponds to $44 \%$ of the slack length. Participants were taking a quiet, upright standing posture during spring attachment (see Figure 3). The spring pretension was calculated as the difference between the tensioned spring length (measured with a millimetre-ruler), and the slack length (constant spring characteristic).

\subsection{Experimental Setup}

Participants walked barefoot on a split-belt treadmill (V-Gait Dual Belt, Motekforce Link, The Netherlands) with integrated force plates to measure ground reaction forces (GRFs) and center of pressure (COP) at a frequency of $2000 \mathrm{~Hz}$ for each belt. Walking kinematics and spring extension were measured with a motion capture system including ten cameras (Bonita B10, VICON, UK, measurement frequency $100 \mathrm{~Hz}$ ) tracking a set of 17 passive reflective markers. Markers were placed bilaterally on the fifth metatarsals, the lateral maleoli, and the lateral epicondyles and greater trochanters of the femur. Additional markers were placed on the left and right acromion and on the spinuous process of the seventh cervical vertebra. To track the motion of the springs, six additional markers were placed anteriorly at the upper and lower spring attachment points, and anteriorly at the distal end of the textile thigh brace (see Figure 3). Electrical muscle activity was measured with 14 wireless electromyography (EMG) transmitters (aktos nano, myon AG, Switzerland) at $2000 \mathrm{~Hz}$ and recorded synchronously with kinematic and force data using Nexus 2.5 software (VICON, UK). Hydrogel/Ag/AgCl surface electrodes (Kendall Arbo H124SG, Covidien, Ireland) were placed bilaterally in a bipolar configuration over seven muscles - gluteus maximus (GMAX), rectus femoris (RF), biceps femoris long head (BF), vastus medialis (VAS), gastrocnemius medialis (GAS), soleus (SOL) and tibialis anterior (TA) - following standard procedures (Surface EMG for the Non-Invasive Assessment of Muscles (SENIAM Project, 2018)). 


\subsection{Experimental Protocol}

Two different spring setups - a single elastic layer, or low stiffness spring (LO) and two elastic layers, or high stiffness spring (HI) - were compared against a reference condition in which no spring $(\mathrm{NO})$ was attached, resulting in a total of three experimental conditions. Two additional conditions in which the same springs were used at a reduced pretension of $2 \mathrm{~cm}$ or $25 \%$ of the slack length were tested within the same visit for a separate study. Participants completed the five conditions in a randomized order to control for temporal effects. Each measurement started with $10 \mathrm{~s}$ of quiet standing in a neutral upright posture, followed by a non-random sequence of level walking at speeds of $0.5,0.9,1.3,1.7$ and $2.1 \mathrm{~m} / \mathrm{s}$ for $70 \mathrm{~s}$ each, ending with another $10 \mathrm{~s}$ of quiet standing. At the beginning of each experimental session, participants completed one disregarded run with the HI stiffness spring to acclimatize and to adjust garments if needed before the start of the core experiment.

\subsection{Data Processing}

Marker kinematics and GRF/COP measurements were used to calculate joint angles and moments using biomechanics simulation software (OpenSim 3.3) with a simplified and individually scaled version of the Gait 2354 lower extremity model (Anderson and Pandy, 1999) and an inverse dynamics approach. The hip center of rotation was approximated by the greater trochanter head following Weinhandl and O'Connor (2010). Spring moments with respect to the hip center of rotation were calculated based on instantaneous spring lengths and lever arms and the previously determined linear spring stiffness. During the experiment, changes in spring length were tracked frame-by-frame by means of the distance between markers placed at the upper and the lower attachment of the spring. The moment lever arm with respect to the hip center of rotation was approximated by the perpendicular distance between a marker on the trochanter head and the line connecting the upper and the lower spring marker in the sagittal plane (see Figure 3 and Supplementary Information for more details).

Energy storage and return phases were identified stride-by-stride as periods between the onset of negative spring power, the zero-crossing between negative and positive spring power, and the end of positive spring power (see Figure 1). Periods with power magnitudes within the $95 \%$ confidence interval around zero power were considered to belong neither to storage nor to return phase. Early and late mid stance were identified for each stride as 
first and second half of the period between weight-acceptance and push-off as defined by Winter (1987).

210 The added mass of the wearable demonstrator (in total, $250 \mathrm{~g}$ per leg) was considered negligible with respect to the inertial and gravitational effects on participants' walking patterns and disregarded in calculations.

EMG raw data were filtered with a zero-phase bandpassfilter (ParksMcClellan, passband between 20 and $400 \mathrm{~Hz}$ ), rectified, and the root-mean215 square value over a moving window of $50 \mathrm{~ms}$ was determined. Raw marker data used for spring moment calculations and results from inverse kinematics and dynamics calculations were filtered with a zero-phase lowpass filter (Parks-McClellan, $14 \mathrm{~Hz}$ cutoff frequency).

Vertical GRF data was used to segment measurements into individual strides. For this purpose, a threshold of $40 \mathrm{~N}$ was used to differentiate between swing and stance phases after a zero-phase lowpass-filter (ParksMcClellan, $22 \mathrm{~Hz}$ cutoff frequency) was applied. All measured time-series were averaged over all strides and over the left and right leg to determine mean and standard error curves for each participant. The first five strides and the last step at each speed were disregarded to avoid capturing transient effects, leaving at least 50 strides for each participant in every condition to be analyzed.

\subsection{Statistical Analysis}

For all of the measured signals, arithmetic means were calculated over functional parts of the gait cycle (either spring-related or kinematic, see Figure 1). A two-way repeated-measures model with within-subject factors "spring stiffness" and "speed", an interaction term between the two withinsubject factors, and a random factor "participant" was used to perform an analysis of variance (ANOVA). The within-subject factors could each take three levels, or $0 \mathrm{~N} / \mathrm{m}$ ("NO"), $801 \mathrm{~N} / \mathrm{m}$ ("LO") and $1602 \mathrm{~N} / \mathrm{m}$ ("LO") for spring stiffness, and $0.5 \mathrm{~m} / \mathrm{s}, 0.9 \mathrm{~m} / \mathrm{s}$ and $1.3 \mathrm{~m} / \mathrm{s}$ for speed. Two additional levels for speed $(1.7 \mathrm{~m} / \mathrm{s}$ and $2.1 \mathrm{~m} / \mathrm{s})$ were included for analyses presented in the Supplementary Information. When Mauchly's test indicated that the assumption of sphericity was violated, a Greenhouse-Geiser correction was applied. If the ANOVA indicated a significant main effect for the factor spring stiffness in absence of a significant interaction with speed, we proceeded to perform post hoc tests for differences between the marginal means across all speeds of the LO and HI spring stiffness levels to the zero-stiffness NO condition by means of paired t-tests. If a significant interaction between 
spring stiffness and speed was found, we instead discussed the nature of the interaction by visual inspection and individual paired t-tests at the different levels of speed. To control the familywise error rate over multiple tests, we have followed the Holm-Bonferroni procedure. Effects were generally considered significant if corrected p-values were lower than 0.05.

\section{Results}

Effects of the springs were found to be consistent over all tested walking speeds, but of decreasing relative magnitude as speed increased. For the sake of clarity, we present only the lower three $(0.5,0.9,1.3 \mathrm{~m} / \mathrm{s})$ out of the five speeds tested in the main paper. Nevertheless, the results for the higher walking speeds are provided in the Supplementary Information.

\subsection{Effects on Hip Joint}

Spring Energy Storage Phase

We hypothesized that the spring would increase the mean positive biological hip power as the spring is extended and stores energy (Hypothesis I). An ANOVA of mean positive biological hip power during the energy storage phase revealed no differences between the group means of the NO, LO and HI conditions $(F(2,16)=0.04, p=0.93$, see Figure 4.D). Similarly, no effect of the spring on total hip power during energy storage phase was observed $(F(2,16)=0.25, p=0.72)$.

As shown in Figure 5, the peak hip extension angle and the mean extension velocity during energy storage phase were not affected by the spring $(F(2,16)=0.20, p=0.78$, and $F(2,16)=0.31, p=0.65$, respectively $)$. In addition, no effect of the spring on the mean EMG activity of GMAX $(F(2,16)=0.36, p=0.66), \operatorname{BF}(F(2,16)=1.53, p=0.25)$ and $\operatorname{RF}(F=$ $0.42, p=0.62)$ during the energy storage phase was found.

\section{Spring Energy Return Phase}

Further, we hypothesized that the spring would reduce the mean positive biological hip power during energy return phase, and thereby, support hip flexion (Hypothesis II). Our results indicate a significant interaction effect of spring stiffness and speed on the mean positive biological hip power during the energy return phase $(F(4,32)=5.93, p=0.018)$. The HI spring stiffness was associated with reduced mean positive biological hip power compared to NO spring at all speed levels $(0.5 \mathrm{~m} / \mathrm{s}: p=0.049,0.9 \mathrm{~m} / \mathrm{s}: p=0.014,1.3 \mathrm{~m} / \mathrm{s}$ : 
$p<0.001)$. A more pronounced reduction was observed at higher speeds. 280 At the same time, an effect $(F(2,16)=8.10, p=0.005)$ of spring stiffness on the mean total hip power output during energy return phase was found. Mean total hip power increased in the LO and HI spring stiffness conditions compared to NO spring during this phase $(p=0.002$ and $p=0.023)$.

The joint power changes were accompanied by an effect of the spring stiffness on the mean hip flexion velocity $(F(2,16)=6.68, p=0.012$, see Figure 5.D). Increased mean hip flexion velocity was observed for the LO $(p=0.031)$ and HI $(p=0.002)$ stiffness springs.

\subsection{Transfer Effects on Ankle Joint}

\section{Spring Energy Storage Phase}

290 We expected a modulation in the quasi-stiffness of the ankle joint in response to the added stiffness of the hip spring during the energy storage phase. Such a modulation would be reflected in changed EMG activity of GAS, SOL, and TA (see Hypothesis III).

During early mid stance, or approx. the first half of energy storage phase, we found an effect of the spring stiffness on the EMG activity of GAS $(F(2,16)=20.91, p<0.001)$, where increased spring stiffness was associated with reduced muscle activity (LO: $p=0.002$, HI: $p<0.001$ ), and a similar but non-significant trend for $\operatorname{SOL}(F(2,16)=1.88, p=0.20$, see Figure 6). An interaction of spring stiffness and speed on TA activity was observed $(F(4,32)=5.23, p=0.027)$. The increase of TA activity that was found for the HI stiffness spring at $0.5 \mathrm{~m} / \mathrm{s}(p=0.014)$ was not significant at higher speeds. During late mid stance, the activity of SOL was affected by the spring $(F(2,16)=11.97, p=0.003)$, such that increased spring stiffness increased muscle activity (LO: $p=0.008$, HI: $p=0.005$ ). During this phase, no effect of the spring on GAS activity was found $(F(2,16)=2.06, p=0.17)$.

Furthermore, the negative ankle joint power during mid stance showed a trend towards reduced magnitudes with increased spring stiffness (see Figure $7, F(2,16)=1.69, p=0.23)$, as would be expected for the found EMG effects.

No effect of the spring on ankle peak dorsiflexion $(F(2,16)=0.70, p=$ $0.47)$ or mean dorsiflexion velocity $(F(2,16)=1.23, p=0.31)$ was observed (see Figure 5.G and Figure 5.I). 


\section{Spring Energy Return Phase}

The spring had an effect on peak ankle plantarflexion (see Figure 5.H) and associated with increased peak plantarflexion $(F(2,16)=5.90, p=0.021$, LO: $p=0.21$, HI: 0.024) and faster plantarflexion $(F(2,16)=18.53, p<$ 0.001, LO: $p=0.017$, HI: $p=0.001)$ during the energy return phase.

In line with our expectations, no effects of the spring on ankle joint power or muscle activity were found during the energy return phase.

\section{Discussion}

\subsection{Biomechanical Effects of Hip Springs}

Spring Energy Storage Phase

During the energy storage phase, the spring was extended and thereby elastically stored energy. As anticipated, joint angles during energy storage were not affected by the spring condition. However, we did not observe an increase in mean positive biological hip power. Instead, the added hip spring stiffness was likely compensated by a decrease in the dorsiflexion quasistiffness of the ankle joint, as reflected in the reduced GAS/SOL activities elastic loading of the Achilles tendon over which they are both connected to the calcaneus (Ishikawa et al., 2005). Given this serial elastic configuration, an activity reduction in these muscles would also suggest reduced linear force and hence elastic energy storage in the Achilles tendon. Our observation of dicating that more active muscle work was required, likely due to the Achilles tendon providing less passive elastic recoil. From an energy perspective, the hip spring apparently stored proportions of the energy that would normally be stored in the Achilles tendon.

340 Participants compensated for the added parallel stiffness at the hip with a decrease in ankle quasi-stiffness, probably aiming to preserve whole-leg stiffness and energy storage during stance phase. Such a pattern would conceptually agree with previous findings that humans modulate their whole-leg stiffness by muscular adaptation to match their current gait speed (Kim and 345 Park, 2011). 


\section{Spring Energy Return Phase}

During the energy return phase, the spring returned the energy stored during the energy storage phase, except for proportions that were dissipated during mechanical deformation. Regarding kinetics, a moment with respect to the hip joint was induced by the spring that acted in parallel to the biological hip flexion moment.

For the LO spring, our data suggest that the spring primarily augmented the total hip power during flexion (see Figure 4). In contrast to our hypothesis, the mean positive biological hip power was similar to NO spring. The stiffer HI spring, in contrast, indeed reduced the mean positive biological hip power while causing an increase in the mean total hip power that was similar to the LO spring. Mooney and Herr (2016) reported similar findings for the ankle, where the addition of an active ankle exoskeleton increased total ankle power. Our assumption that spring contributions would solely replace biological power is hence contradicted.

Joint angles during energy return phase were affected by the added springs when compared to the NO spring condition (see Figure 5). Notably, increases in hip flexion velocity and peak hip flexion angle were already seen for the LO spring, and were more pronounced for the HI spring. Thus, it seems that kinematic deviations from regular walking were tolerated before joint powers were modulated, as seen in the LO spring condition. Only when the spring stiffness increased further, joint powers were adapted (as in HI). Our experimental data shows a contribution to hip extension quasi-stiffness of 10 $\mathrm{Nm} / \mathrm{rad}$ and $20 \mathrm{Nm} / \mathrm{rad}$ for the LO spring and HI spring, respectively. Consequently, the stiffness threshold to transition from purely kinematic adaptation (as in LO) to the joint power modulation observed for the HI spring could be placed between 10 and $20 \mathrm{Nm} /$ rad for level walking. However, considering the moderate sample size and high process variability of our experiment, we cannot completely rule out that minor power adaptations occurred at lower stiffness, but were not captured by our measurements and statistical analysis.

\subsection{Design Implications}

Our findings highlight the importance of considering all leg joints during the design, simulation and use of spring-like orthoses, even if the orthoses only act on a single joint. Specifically, our assumptions that the biological hip power needs to increase to maintain normal leg kinematics during the energy storage phase of a hip spring and that total hip power would stay 
constant during energy return are contradicted by our results. Design conclusions of hip exoskeleton simulation studies based on these assumptions (e.g. Chen et al. (2019)) should hence be questioned. For the design of springs, it might be more instructive to consider power redistribution to and from elastic structures at all leg joints, instead of the moments the springs induce with respect to the anatomically spanned joints. Similar approaches have been previously applied to simulate the expected energetic benefits of active assistive devices (Dembia et al., 2017).

In our case of an anteriorly placed hip spring, that would mean primarily considering the desired remaining energy storage in the Achilles tendon versus the targeted energy return for hip flexion assistance. Interestingly, Collins et al. (2015) observed a decrease of GAS and SOL activity accompanied by an increase in TA activity during mid stance using a clutched posterior ankle spring, similar to our findings for the anterior hip spring. Hence, springs placed across the ankle and hip might primarily differ with respect to time and duration of energy return (ankle spring having earlier onset and being shorter than the hip), but not necessarily in the way energy is initially stored.

While Sutliff et al. (2008) and Carda et al. (2012) have shown that anterior hip springs can lead to functional benefits for multiple sclerosis and stroke patients, such benefits might consequently extend to populations with limited capacity for elastic energy storage in the Achilles tendon, e.g. due to normal aging (Franz and Thelen, 2016) or injury (Frankewycz et al., 2018). For future work, we hypothesize that an added hip spring could partially replace the lost elastic energy storage capacity and hence improve walking performance. However, clinical trials with functional outcome measures such as the 10-meter- or six-minute-walking-test are needed to evaluate this hypothesis.

\subsection{Study Limitations}

410 Typical quasi-stiffness values of the hip during walking range from $124 \mathrm{Nm} / \mathrm{rad}$ to $516 \mathrm{Nm} / \mathrm{rad}$ (Shamaei et al., 2013). We intentionally did not design our demonstrator to allow exploration of this entire range. Instead, we prioritized a design with minimal mass and bulk as a valid reference for simulations and as a mostly hardware-independent basis for design decision. We also decided to use fixed spring stiffnesses for all participants, instead of individually scaling spring stiffnesses to the biological hip joint quasi-stiffness. This choice was motivated by the fact that the reported model error of individualization 
is on the same order of magnitude as the largest expected difference following scaling in our targeted study population (approx. $30 \%$, Shamaei et al. 420 (2013)).

Furthermore, it remains unclear to which extent our demonstrator increases the load on upper body muscles that maintain postural stability. Potentially, such loading might limit the range of tolerable spring stiffnesses for people with postural deficits. However, a more refined set of garments could allow load transfer to the hip instead of the shoulders, as recently presented by Schmidt et al. (2017).

\section{Conclusion}

In healthy users, a spring attached anteriorly across the hip appears to store and return proportions of the elastic energy that would normally be exchanged with the Achilles tendon during walking. As energy is returned, the spring assists hip flexion, while muscle activity related to ankle plantarflexion around push-off increases.

Our findings strongly highlight the importance of the functional coupling between the hip and the ankle joint during walking for the design of wearable robots and orthoses. The anterior hip springs used in this work caused changes in hip and ankle joint dynamics and calf muscle activities that were very similar to the ones observed when using an ankle spring, particularly during the energy storage phase of the spring. Hence, the anatomical joint around which moments are induced might not be as important as one might first assume. In fact, the decision regarding where to add passive springs at the hip or at the ankle - might be primarily driven by practical considerations pertaining to attachment and comfort as well as temporal aspects. Future design processes should therefore consider energy storage and return phases along the gait cycle, and depart from quasi-static, joint moment-based approaches. We believe that our findings are well-suited to serve as a reference in such designs or simulations, given the low mass and footprint of our demonstrator that introduces continuously quantifiable forces without major changes in body inertia or wearer comfort. Future work should investigate how effects described in this work transfer to clinical target populations and attempt to link biomechanical observations to clinical outcomes. 


\section{Acknowledgements}

This research was supported by the Swiss National Science Foundation through the National Centre of Competence in Research Robotics (NCCR Robotics) and by the Swiss Center for Clinical Movement Analysis (SCMA), Balgrist Campus AG. We would like to thank Charlotte Werner for her support with measurements and data processing, and Andrew Pennycott for constructive criticism of the manuscript.

\section{Conflict of Interest}

The authors declare that there is no conflict of interest regarding the content of this article.

\section{References}

Anderson, F. C. and M. G. Pandy 1999. A dynamic optimization solution for vertical jumping in three dimensions. Computer Methods in Biomechanics and Biomedical Engineering, $2(3): 201-231$.

Blaya, J. A. and H. Herr 2004. Adaptive control of a variable-impedance ankle-foot orthosis to assist drop-foot gait. IEEE Transactions on Neural Systems and Rehabilitation Engineering, 12(1):24-31.

Bregman, D. J. J., J. Harlaar, C. G. M. Meskers, and V. de Groot 2012. Spring-like ankle foot orthoses reduce the energy cost of walking by taking over ankle work. Gait \& Posture, 35(1):148-153.

Cahill, N. M., T. Sugar, Y. Ren, and K. Schroeder 2018. Optimal stiffness design for an exhaustive parallel compliance matrix in multiactuator robotic limbs. Journal of Mechanisms and Robotics, 10(3):031014-1-031014-7.

Carda, S., M. Invernizzi, G. Cognolato, E. Piccoli, A. Baricich, and C. Cisari 2012. Efficacy of a hip flexion assist orthosis in adults with hemiparesis after stroke. Physical Therapy, 92(5):734-739. 
Cavagna, G. A., N. C. Heglund, and C. R. Taylor

1977. Mechanical work in terrestrial locomotion: two basic mechanisms for minimizing energy expenditure. American Journal of PhysiologyRegulatory, Integrative and Comparative Physiology, 233(5):R243-R261.

Chen, W. B., S. Wu, T. C. Zhou, and C. H. Xiong 2019. On the biological mechanics and energetics of the hip joint muscletendon system assisted by passive hip exoskeleton. Bioinspiration \&6 Biomimetics, 14(1):016012-1-016012-14.

Collins, S. H., M. B. Wiggin, and G. S. Sawicki 2015. Reducing the energy cost of human walking using an unpowered exoskeleton. Nature, 522(7555):212-215.

Dembia, C. L., A. Silder, T. K. Uchida, J. L. Hicks, and S. L. Delp 2017. Simulating ideal assistive devices to reduce the metabolic cost of walking with heavy loads. PLOS ONE, 12(7):e0180320.

Farris, D. J. and G. S. Sawicki 2012. The mechanics and energetics of human walking and running: a joint level perspective. Journal of the Royal Society Interface, 9(66):110-118.

Ferris, D. P., Z. A. Bohra, J. R. Lukos, and C. R. Kinnaird 2006. Neuromechanical adaptation to hopping with an elastic ankle-foot orthosis. Journal of Applied Physiology, 100(1):163-170.

Frankewycz, B., A. Penz, J. Weber, N. P. da Silva, F. Freimoser, R. Bell, M. Nerlich, E. M. Jung, D. Docheva, and C. G. Pfeifer 2018. Achilles tendon elastic properties remain decreased in long term after rupture. Knee Surgery, Sports Traumatology, Arthroscopy, 26(7):20802087.

Franz, J. R. and D. G. Thelen

2016. Imaging and simulation of achilles tendon dynamics: Implications for walking performance in the elderly. Journal of Biomechanics, 49(9):14031410 .

Hobara, H., K. Inoue, T. Muraoka, K. Omuro, M. Sakamoto, and K. Kanosue 2010. Leg stiffness adjustment for a range of hopping frequencies in humans. Journal of Biomechanics, 43(3):506-511. 
Ishikawa, M., P. V. Komi, M. J. Grey, V. Lepola, and G. P. Bruggemann 2005. Muscle-tendon interaction and elastic energy usage in human walking. Journal of Applied Physiology, 99(2):603-608.

Kim, S. and S. Park

2011. Leg stiffness increases with speed to modulate gait frequency and propulsion energy. Journal of Biomechanics, 44(7):1253-1258.

Latash, M. L. and Zatsiorsky, V. M.

1993. Joint stiffness: Myth or reality? Human Movement Science, 12(6):653-692.

Lenzi, T., M. C. Carrozza, and S. K. Agrawal

2013. Powered hip exoskeletons can reduce the user's hip and ankle muscle activations during walking. IEEE Transactions on Neural Systems and Rehabilitation Engineering, 21(6):938-948.

Mooney, L. M. and H. M. Herr

2016. Biomechanical walking mechanisms underlying the metabolic reduction caused by an autonomous exoskeleton. Journal of Neuroengineering and Rehabilitation, 13.

Nasiri, R., A. Ahmadi, and M. N. Ahmadabadi 2018. Reducing the energy cost of human running using an unpowered exoskeleton. IEEE Transactions on Neural Systems and Rehabilitation Engineering, 26(10):2026-2032.

Neptune, R. R., F. E. Zajac, and S. A. Kautz 2004. Muscle force redistributes segmental power for body progression during walking. Gait \& Posture, 19(2):194-205.

Rouse, E. J., R. D. Gregg, L. J. Hargrove, and J. W. Sensinger 2013. The difference between stiffness and quasi-stiffness in the context of biomechanical modeling. IEEE Transactions on Biomedical Engineering, 60(2):562-568.

Schmidt, K., J. E. Duarte, M. Grimmer, A. Sancho-Puchades, H. Wei, C. S. Easthope, and R. Riener

2017. The Myosuit: Bi-articular anti-gravity exosuit that reduces hip extensor activity in sitting transfers. Frontiers in Neurorobotics, 11(57). 
SENIAM Project

2018. Available at www.seniam.org, retrieved 15.12.2017.

Shamaei, K., M. Cenciarini, A. A. Adams, K. N. Gregorczyk, J. M. Schiffman, and A. M. Dollar

2015. Biomechanical effects of stiffness in parallel with the knee joint during walking. IEEE Transactions on Biomedical Engineering, 62(10):23892401.

Shamaei, K., G. S. Sawicki, and A. M. Dollar

2013. Estimation of quasi-stiffness of the human hip in the stance phase of walking. PLoS ONE, 8(12):e81841.

Shepherd, M. K. and E. J. Rouse 2017. Design of a quasi-passive ankle-foot prosthesis with biomimetic, variable stiffness. In 2017 IEEE International Conference on Robotics and Automation (ICRA), Pp. 6672-6678.

Simonsen, E. B., K. L. Cappelen, R. Skorini, P. K. Larsen, T. Alkjaer, and P. Dyhre-Poulsen

560 2012. Explanations pertaining to the hip joint flexor moment during the stance phase of human walking. Journal of Applied Biomechanics, 28(5):542-50.

Sutliff, M. H., J. M. Naft, D. K. Stough, J. C. Lee, S. S. Arrigain, and F. A. Bethoux

2008. Efficacy and safety of a hip flexion assist orthosis in ambulatory multiple sclerosis patients. Archives of Physical Medicine and Rehabilitation, 89(8):1611-1617.

Walsh, C. J., K. Endo, and H. Herr 2007. A quasi-passive leg exoskeleton for load-carrying augmentation. International Journal of Humanoid Robotics, 04(03):487-506.

Weinhandl, J. T. and K. M. O'Connor

2010. Assessment of a greater trochanter-based method of locating the hip joint center. Journal of Biomechanics, 43(13):2633-2636.

Winter, D. A.

575 1987. The Biomechanics and Motor Control of Human Gait. Waterloo, Canada: University of Waterloo Press. 


\section{Figures}

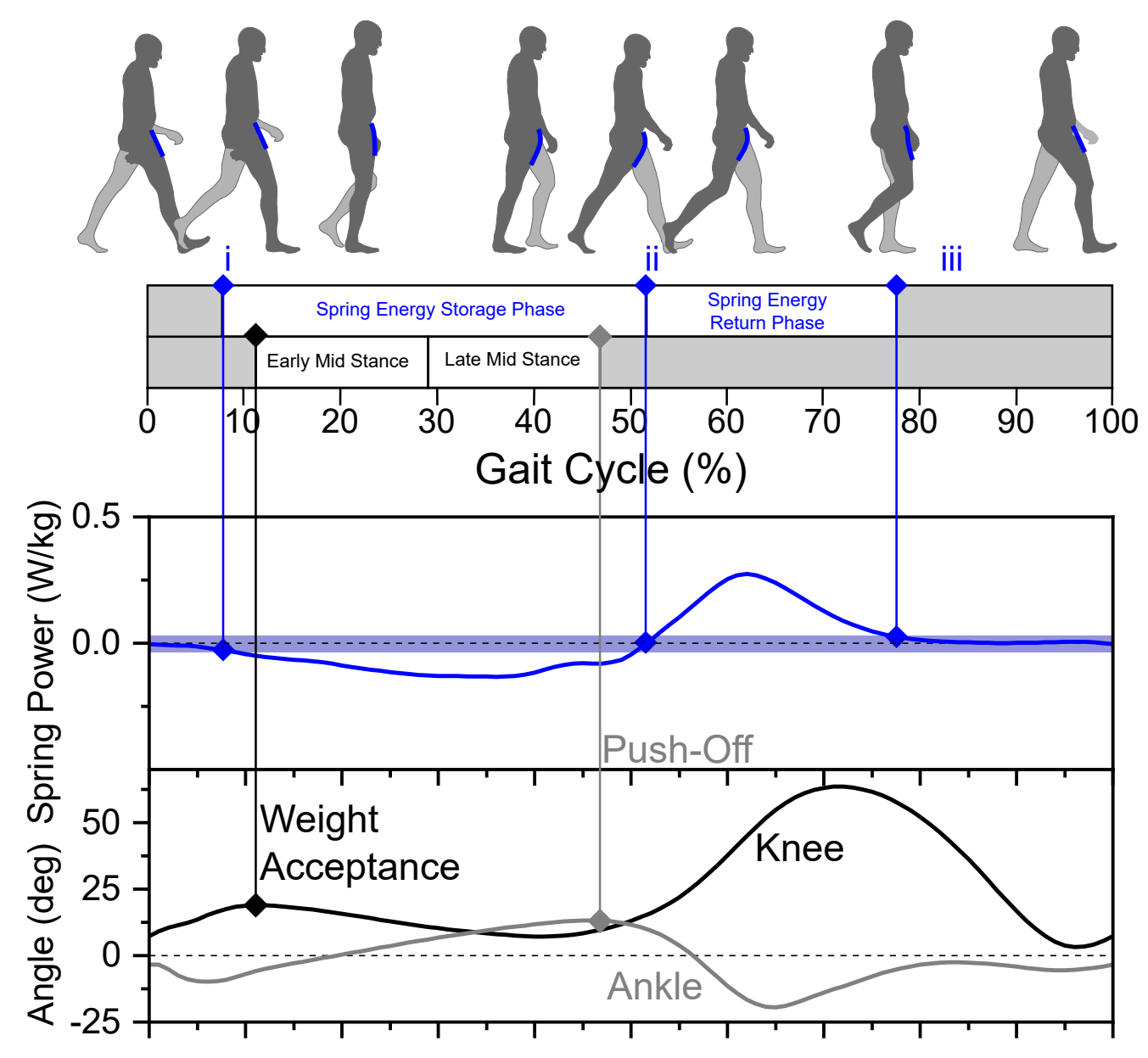

Figure 1: Schematic overview of the functional phases of a passive spring anteriorly crossing the hip joint (blue print) and gait phases relevant for elastic energy storage in the Achilles tendon adapted from Winter (1987) (black print). We define the gait cycle from heel strike $(0 \%)$ to subsequent heel strike of the same foot (100\%). For the anterior hip springs, two main phases can be identified: "spring energy storage phase", defined as the period of negative spring power, and "spring energy return phase", defined as the period of positive spring power. The period between weight acceptance and push-off is divided into early and late mid stance. 


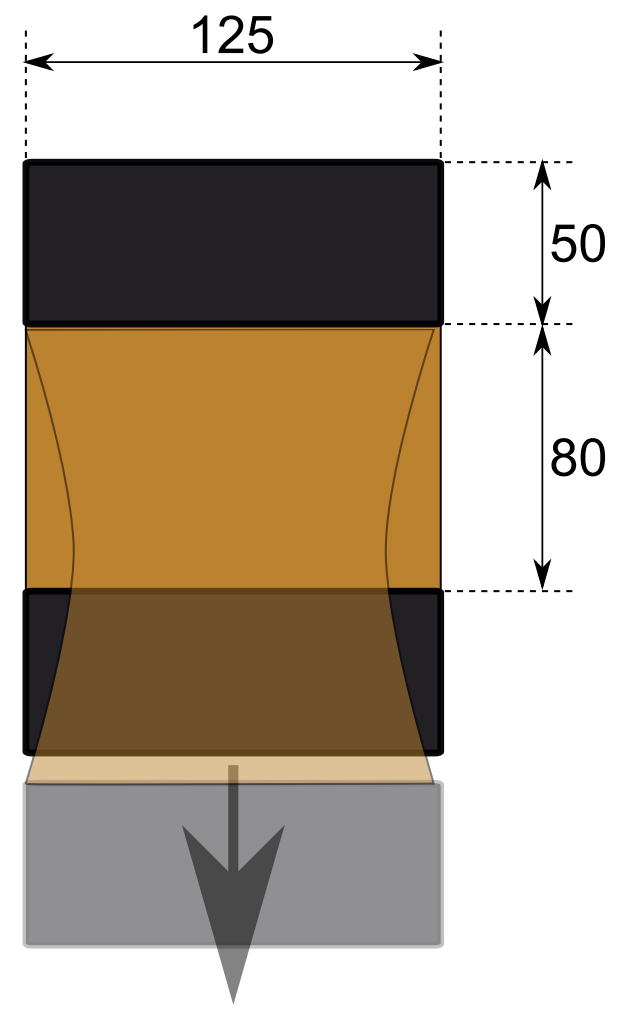

Figure 2: Sketch of the geometry of the spring elements placed anteriorly across the hip. Dimensions are given in millimetres. 

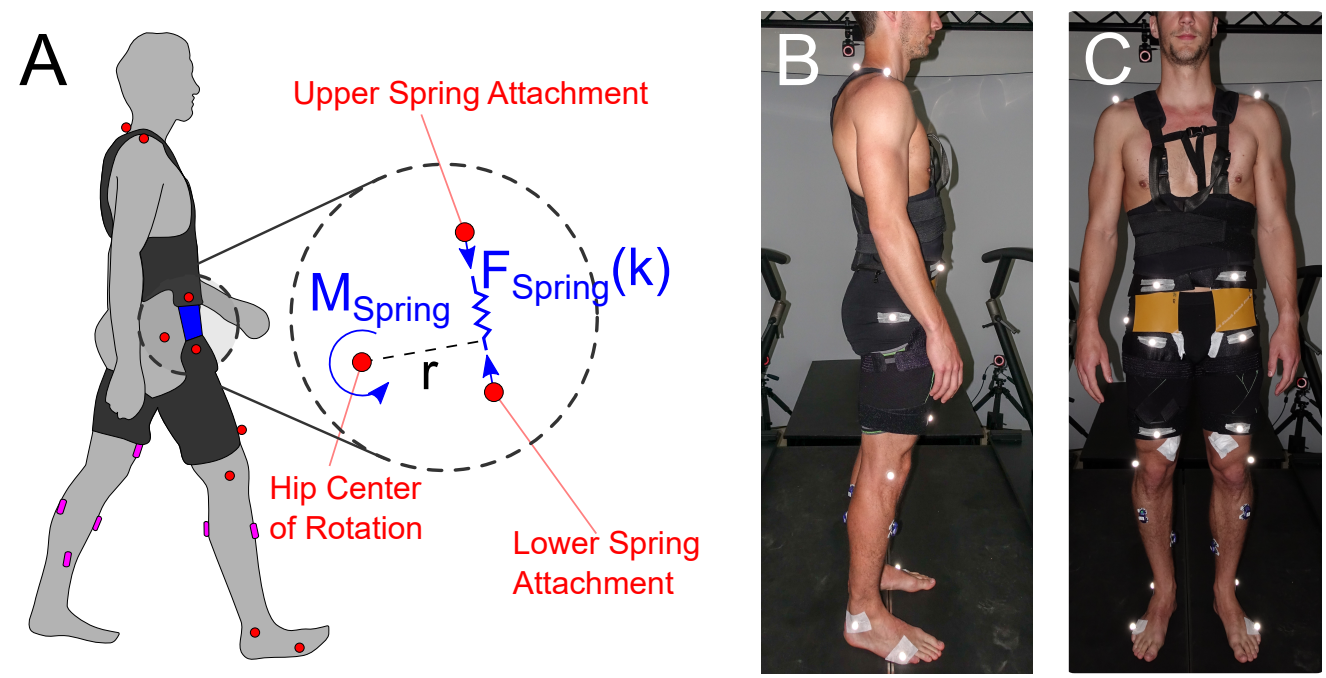

Figure 3: (A) Illustration of the wearable demonstrator setup (dark grey), markers (red) and EMG sensors (magenta) worn by study participants. The anteriorly placed spring (blue) induces a flexion moment with respect to the hip joint center of rotation which is approximated by the trochanter head marker. The moment magnitude depends on the instantaneous spring elongation (distance between the lower and the upper spring attachment marker), the perpendicular lever arm $\mathrm{r}$, and the previously determined linear spring constant k. (B) Sagittal plane picture of a participant. (C) Frontal plane picture of a participant. 

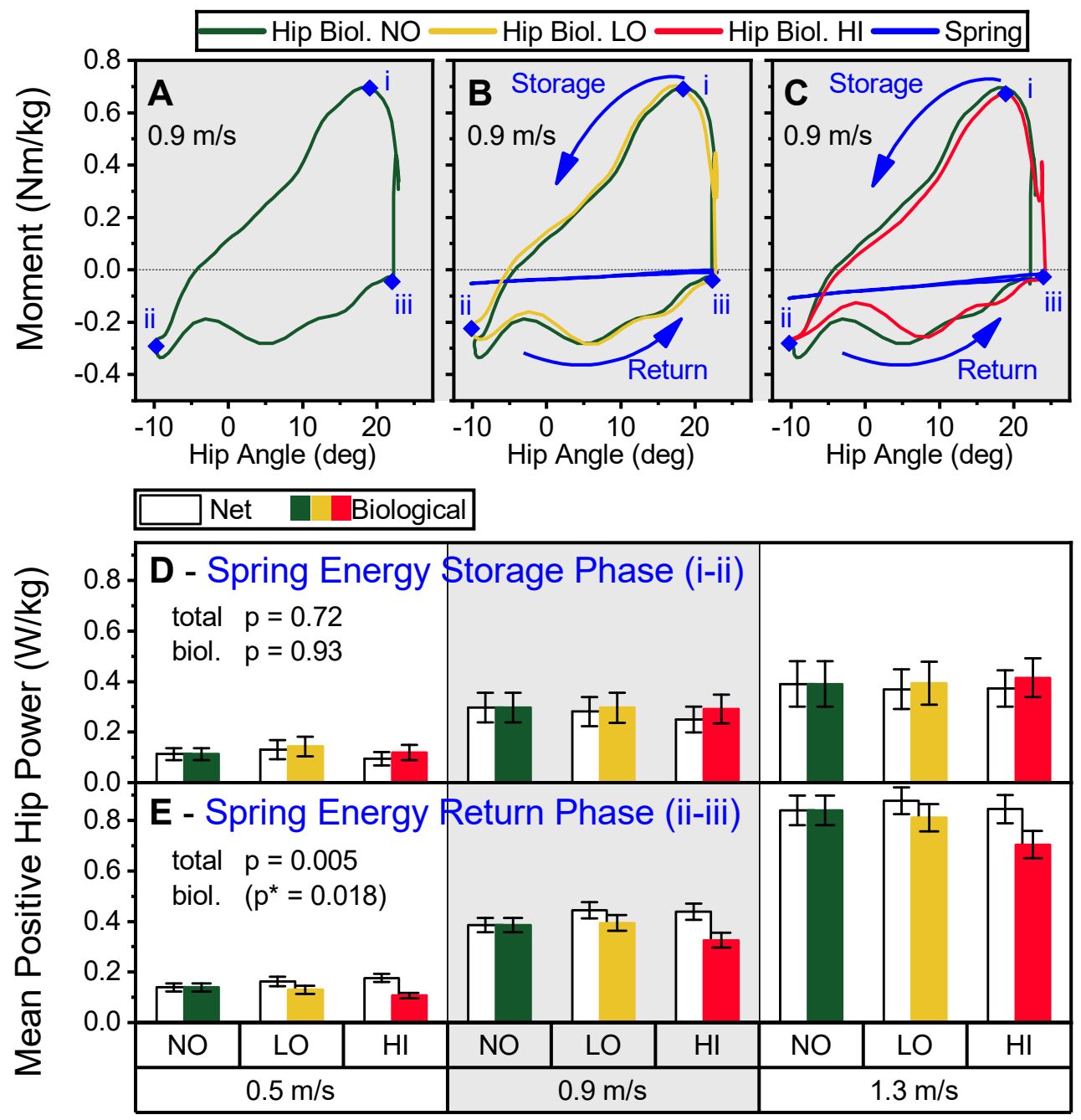

Figure 4: Panels A-C show the mean biological hip moment plotted against mean hip angle at a walking speed of $0.9 \mathrm{~m} / \mathrm{s}$. The moment-angle curve when wearing the LO stiffness spring (B, yellow) and the HI stiffness spring ( , red), are shown against the reference with NO spring (dark green). The two main movement arcs of the hip joint are marked with roman numbers i to ii (energy storage phase) and ii to iii (energy return phase). Panels D (loading) and E (support) show mean total (open bars) and biological (colored bars) positive hip power values during the respective phases at $0.5 \mathrm{~m} / \mathrm{s}, 0.9 \mathrm{~m} / \mathrm{s}$ and 1.3 $\mathrm{m} / \mathrm{s}$. P-values are the result of an ANOVA across the three spring conditions and three speeds for a main effect of the spring condition on mean total and mean biological power, respectively. The value in parentheses marked as $\mathrm{p}^{*}$ indicates a significant interaction effect between spring stiffness and speed on the mean positive biological hip power during energy return phase. 


\section{A - Storage B - Return}

\section{$\mathrm{v}=0.9 \mathrm{~m} / \mathrm{s}$}

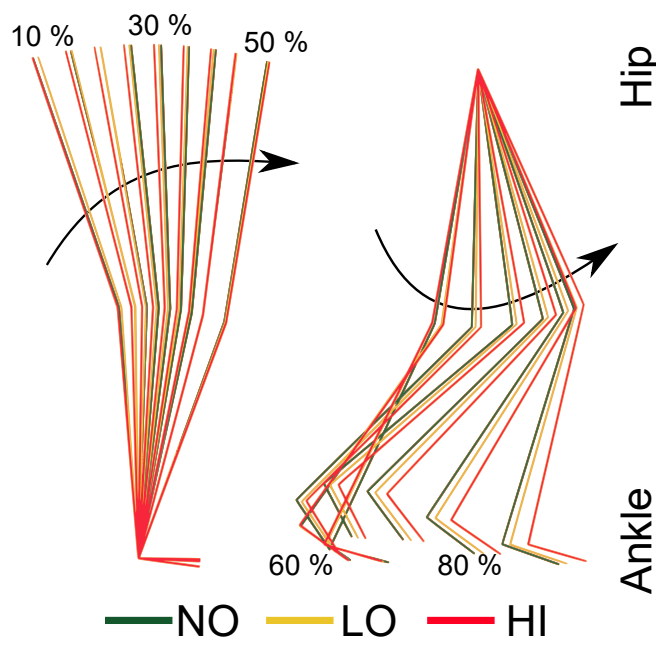

Numbers are (\%) Gait Cycle
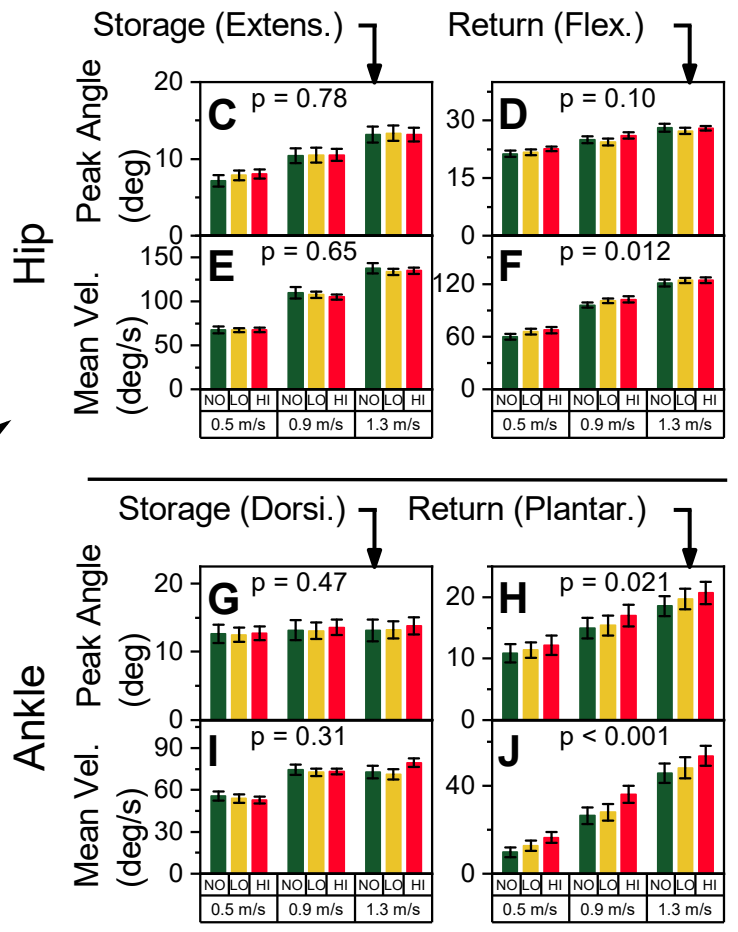

Figure 5: Panels A and B show stick-figures of the mean walking kinematics at $0.9 \mathrm{~m} / \mathrm{s}$ during energy storage phase and energy return phase. Green sticks depict reference kinematics (NO spring), yellow LO spring stiffness kinematics, and red HI stiffness kinematics. Panels C-J show peak angles and mean velocities over energy storage and energy return phase for the hip and ankle joint. Kinematics do not differ between NO, LO and HI conditions during energy storage phase. However, during energy return phase, differences are observed. P-values are the result of ANOVAs across the three spring conditions and three speeds for a main effect of the spring condition on the respective joint angle or velocity. 


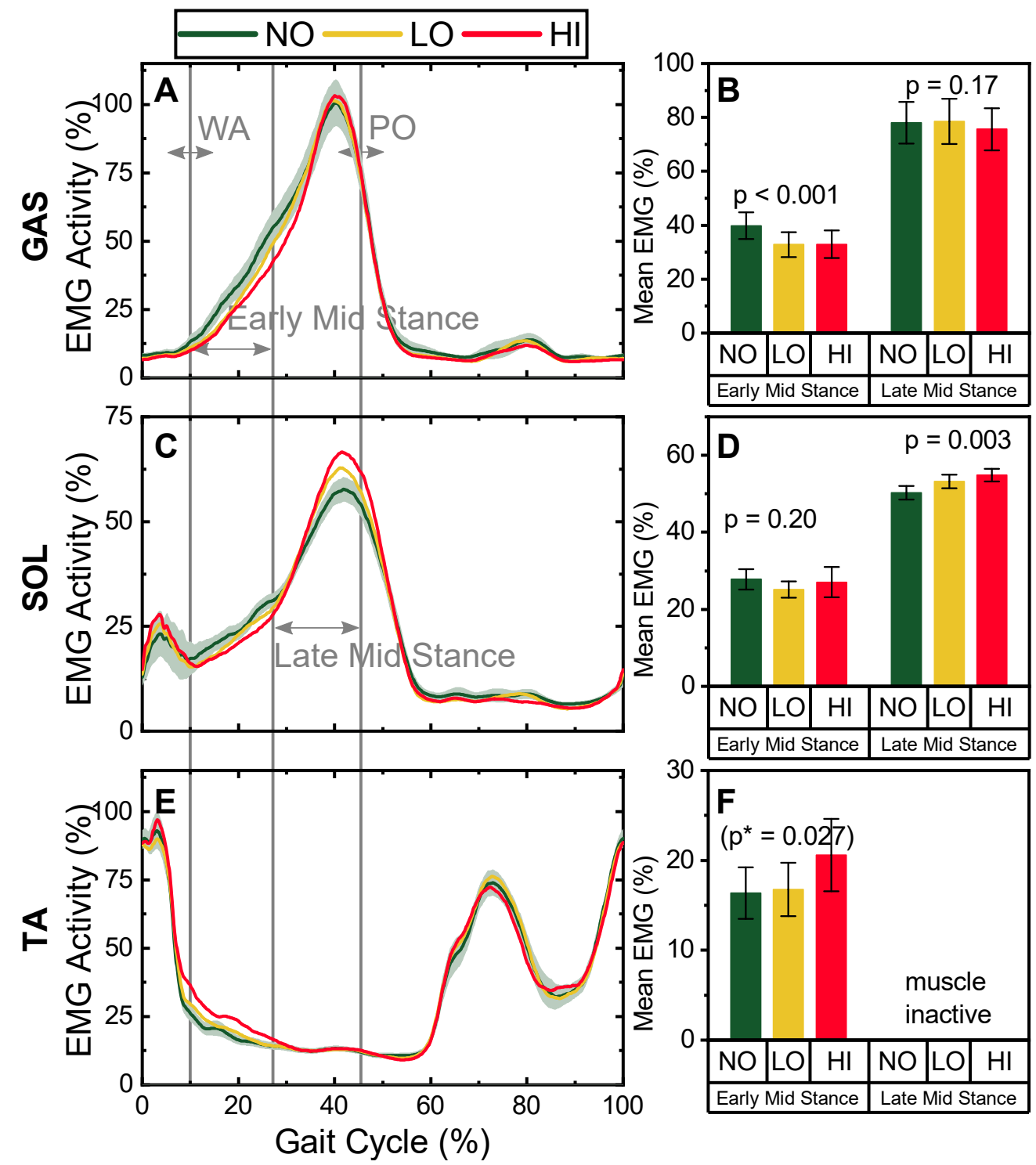

Figure 6: Panels A,C,E show the EMG activity of ankle plantarflexor (GAS, SOL) and dorsiflexor muscles (TA) over one gait cycle at a walking speed of $0.9 \mathrm{~m} / \mathrm{s}$ for the three spring stiffnesses (NO, LO, HI). Panels B,D,F show the mean EMG activity over early and late mid stance for NO, LO and HI spring stiffness conditions at $0.9 \mathrm{~m} / \mathrm{s}$. P-values are the results of ANOVAs across the three spring conditions and three speeds for a main effect of the spring condition on the respective muscle activity in early or late mid stance. The value in parentheses marked as $\mathrm{p}^{*}$ indicates a significant interaction effect between spring stiffness and speed on the activity of TA during early mid stance. 


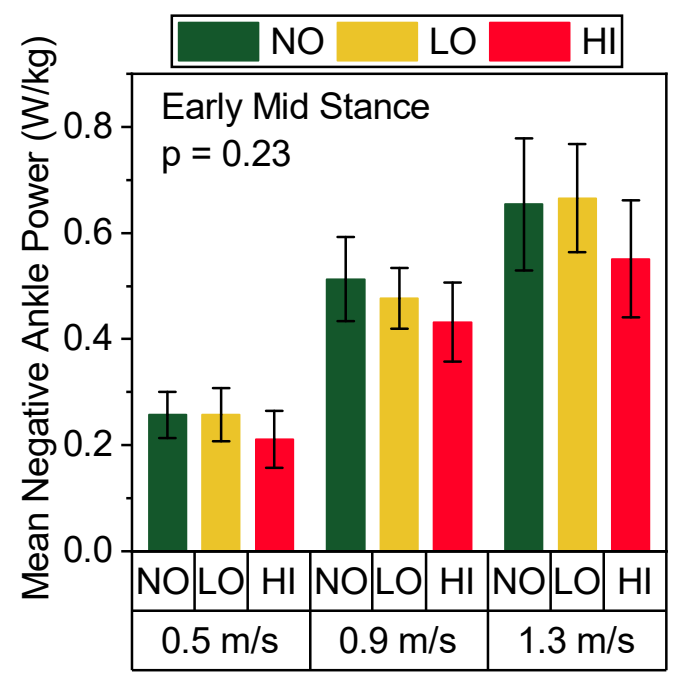

Figure 7: Mean negative ankle power during mid stance for NO spring (green, reference), LO stiffness spring (yellow) and HI stiffness spring (red), at walking speeds of 0.5, 0.9 and $1.3 \mathrm{~m} / \mathrm{s}$. While a tendency towards reduced negative power, i.e. less energy dissipation at the ankle during mid stance is observed, the effect does not reach the significance threshold $(\mathrm{p}=0.23)$. However, it is consistent with significant effects on EMG activity as presented in Figure 6. 